\title{
COMMUNICATION
}

\section{Gestion d'extractions dentaires chez un patient présentant un lymphome $B$ diffus à grandes cellules du sinus maxillaire.}

\author{
Dulong $A^{1,2,3}$, Trensz $P^{4}$, Weingertner $N^{5}$, Herbrecht $R^{4,6}$, Lutz JC ${ }^{3,6}$, Bornert F $F^{2,7}$ \\ 1. UFR d'Odontologie - Université de Reims \\ 2. Unité de Pathologie-Chirurgie buccale, Pôle de Médecine et de Chirurgie bucco-dentaires - HUS Strasbourg \\ 3. Service de Chirurgie Plastique, Esthétique, Reconstructrice, Maxillo-Faciale et Stomatologie - HUS Strasbourg \\ 4. Département d'Hématologie, Pôle d'Oncologie-Hématologie - HUS Strasbourg \\ 5. Département de Pathologie - HUS Strasbourg \\ 6. Faculté de Médecine - Université de Strasbourg \\ 7. Faculté de Chirurgie Dentaire - Université de Strasbourg
}

\section{Introduction}

Les lymphomes non hodgkiniens $(\mathrm{LNH})$ représentent environ $2 \%$ et la 2ème catégorie de tumeurs malignes dans la bouche, en terme de fréquence, après les carcinomes épidermoïdes $(1,2)$. Ils peuvent se présenter dans des localisations nodales ou extra-nodales et notamment au niveau du sinus maxillaire. Un bilan buccodentaire doit être réalisé avant toute thérapeutique anti-cancéreuse.

\section{Observation}

Quatre semaines après l'avulsion des dents 14-15, une prolifération gingivale a permis de révéler un lymphome $B$ diffus à grandes cellules du sinus maxillaire droit chez un homme de 69 ans. Il est alors adressé en chirurgie orale par l'onco-hématologie pour procéder aux avulsions des dents 13, 16 et 17, prises dans la tumeur sinusienne, très douloureuses et mobiles et associées à des difficultés d'alimentation avec perte de 10 $\mathrm{kg}$ en 1 mois. L'examen clinique montre une volumineuse lésion en provenance du sinus. L'OPT retrouve une lésion radioclaire étendue des dents 17 à 12 apparaissant « flottantes ». Le scanner objective une lésion du sinus maxillaire droit faisant saillie au niveau des fosses nasales et des tissus mous périmaxillaires avec lyse des parois mésiale, antérieure et du processus alvéolaire. Les avulsions sont repoussées et les douleurs traitées de manière symptomatique en attendant de revoir le patient après la première cure de chimiothérapie par le protocole R-CHOP + méthotrexate. Trois semaines plus tard la masse a nettement diminué et la NFS étant compatible avec un geste, les avulsions sont réalisées sans particularité. A 15 jours, la cicatrisation est en bonne voie, les douleurs ont totalement disparu et l'alimentation est quasi-normale. Le bilan TEP-scan fait après la 4ème cure de chimiothérapie objective une régression totale de la lésion.

\section{Discussion}

Réaliser des avulsions au sein d'une masse en pleine expansion peut exposer à certaines difficultés : problème d'anesthésie, risque hémorragique, impossibilité de procéder à un curetage ou réaliser des sutures sur des tissus totalement modifiés par l'envahissement tumoral ; le patient peut aussi présenter des anomalies de la NFS. D'autre part, on ne sait pas, dans notre cas, si les avulsions dentaires initiales ont favorisé ou non l'expansion rapide du lymphome au niveau du sinus maxillaire ? II existe peu de cas décrits dans la littérature d'avulsions de dents en contact direct avec un lymphome. Néanmoins, la prudence incite à temporiser ces avulsions dans la mesure où la réponse thérapeutique est souvent très rapide et spectaculaire.

\section{Conclusion}

Bien que peu fréquent dans la bouche, les LNH de type B doivent être évoqués face à des douleurs et/ou mobilités ne cédant pas après un traitement bucco-dentaire adapté. Le diagnostic repose sur l'anatomopathologie et le traitement sur la chimiothérapie incluant du Rituximab. Le taux de survie est de plus de $50 \%$ à 5 ans. Bien que la mise en état de ma cavité buccale soit un prérequis à ces thérapeutiques, il est parfois préférable, en concertation avec l'hématologue, de conserver de manière temporaire certaines dents compromises en attendant la réduction tumorale et de meilleures conditions locales et générales avant de pratiquer un geste chirurgical bucco-dentaire.

dulong.arnaud@orange.fr

(C) The authors, published by EDP Sciences. This is an Open Access article distributed under the terms of the Creative Commons Attribution License 4.0 (http://creativecommons.org/licenses/by/4.0/). 